\title{
Review of complex adaptive systems in nursing practice
}

\author{
J. Kiviliene ${ }^{1}$, A. Blazeviciene ${ }^{2}$ \\ Lithuanian University of Health Sciences, Faculty of Nursing, Kaunas, Lithuania \\ ${ }^{1}$ Corresponding author \\ E-mail: ${ }^{1}$ juste.kiviliene@gmail.com, ${ }^{2}$ aurelija.blazeviciene@lsmuni.lt \\ Received 6 November 2019; received in revised form 16 December 2019; accepted 23 December 2019 \\ DOI https://doi.org/10.21595/chs.2019.21169
}

Check for updates

Copyright (C) 2019 J. Kiviliene, et al. This is an open access article distributed under the Creative Commons Attribution License, which permits unrestricted use, distribution, and reproduction in any medium, provided the original work is properly cited.

\begin{abstract}
This article describes the most typical characteristics of complex adaptive systems (CAS) theory and how it works in healthcare organizations and nursing practice. Complexity science belongs to newest systems thinking, which explore complex systems by focusing on the system components, relationships and interconnections rather than on the individual components themselves. The nurse must therefore always keep in mind the larger picture when caring for the individual patient and expect to be disturbed by unexpected possibilities. The incorporation of complexity science concepts into the practice of nursing leadership encourages new ways of thinking about organizations and is a natural model for the ideals and expected results of skilled nursing. CAS theory will take a permanent place in the healthcare organizations activities, improve nursing processes and create a better work environment for nursing and job satisfaction.
\end{abstract}

Keywords: healthcare, organizations, nurse, theory.

\section{Introduction}

The principles of nursing practice describe what everyone, from nursing staff to patients, can expect from nursing. However, there are a lot of questions in real life. Clinical and organizational procedures are becoming increasingly complex in today's health systems. Various suppliers, new technologies, innovations and growing knowledge ubiquity all lead to the idea of health care as a complex system [1]. So why does it work seamlessly sometimes and not so well on other time? It could be, that in one-time team creates peak level of creativity and then the same team is disorganized on the other day. These are just a few problems which face nursing team members. Increasingly complex adaptive systems (CAS) theory coming in the nursing practice with lots of researches and gives us explanations for why plans, strategies may not work and becomes a significant impact in the health services structures and processes. For instance, once healthcare team members (i.e., nurses) increase their focus on connections, diversity, information flow and creative adaptation promotion the self-organization starts to emerge. For example, variables that increase the flow of information through nursing process and promote timely nursing can improve patient outcomes, while barriers can lead to errors and adverse events [2]. Complexity science builds on the rich tradition in nursing practice that views patients and nursing care from a systems perspective [3]. CAS theory, as a way of understanding organizations and communities, has been considered as an approach within healthcare management rather than as a nursing tool [4]. The health system and the profession of nursing can be viewed as CAS, and when done so, new insight can be gained.

\section{Data source}

A bibliographic search was undertaken to retrieve articles published using the following databases: PubMed $(\mathrm{C}$ and Google Scholar $\subset$. We chose articles in English and published since 2005 . Once the articles were identified $(n=24000)$, duplicates were excluded, the title and the abstract had to contain the following keywords: healthcare, organizations, nurse, theory and after observant analysis articles of interest were selected $(n=16)$. The aim was to identify the evidence in the literature that shows the advantages of complex system approach in healthcare organizations 
and nursing practice.

\section{Main features of complex adaptive systems theory}

It is written in the book "Handbook of Systems and Complexity" that complexity thinking is a change of mindset away from understanding the whole from an understanding of its parts (the Newtonian approach) to an appreciation that the whole is different and less than the sum of its parts. The parts are seen in isolation and exhibit different properties than those seen in the sense of the whole [5]. A CAS is a collection of individual agents (components) who are free to act in ways that are not always entirely predictable and interconnected [3]. It also has distinctive characteristics. Michel Baranger states the most typical ones:

1) Complex systems contain many constituents interacting nonlinearly.

2) The constituents of a complex system are interdependent.

3) A complex system possesses a structure spanning several scales.

4) A complex system is capable of emerging behavior.

5) Complexity involves an interplay between chaos and non-chaos.

6) Complexity involves an interplay between cooperation and competition [6].

CAS theory is important in social healthcare context, which involves the interaction between professionals, patients and the organizational systems in care delivery. Complexity theory may provide an explanatory framework to explain the complexities inherent in implementation in social healthcare contexts [7].

We would like to discuss how complex adaptive systems works in healthcare organizations and nursing practice.

\section{Complex adaptive systems in healthcare organizations}

Complexity science belongs to newest systems thinking, which explore complex systems, also called CAS, by focusing on the system components, relationships and interconnections rather than on the individual components themselves. Complexity science was developed as a theoretical framework in healthcare to better understand complex situations [8]. Interest in ever-growing body of scientific knowledge in complex systems will be presented in the following section.

Ellis B. performed empirical research on quality improvement through case studies of the implementation of clinical governance arrangements in two primary care organizations. She has described developments in primary care informatics and the trend towards federated models of practice and has introduced the concepts of clinical governance and CAS. Clinical management programs have been possible because of developments in the use and functionality of electronic care records, principally in primary care. However, "whole system" thinking and interdisciplinary coordination are equally important enablers. CAS principles provide a framework that enables insight into how those operating within social networks communicate with each other to determine actions that manage their most relevant concerns [9].

Another study explores the implementation experience of nine primary care practices becoming patient-centered medical homes (PCMH) as part of the New Hampshire Citizens Health Initiative Multi-Stakeholder Medical Home Pilot. The purpose of investigation was to apply CAS theory and relationship-centered organizations theory to explore how nine diverse primary care practices in New Hampshire implemented the PCMH model and to offer insights for how primary care practices can move from a structural PCMH to a relationship-centered PCMH. The process of moving from changing the structures to changing the culture of the practice required reframing the approach to implementation of these structures through constant conversation, relationship building, and trust. It is in this framework that understanding primary care practices as CAS is useful. As agents are engaged in this interdependent process of caring for a patient, they interact in nonlinear ways (i.e., through meetings and team building) yielding emergent properties, such as a culture of collaborative care. As these relational routines and patterns start to form a new way 
of thinking about team-based care (i.e., self-organization), the practice starts to look more like a relationship-centered PCMH [10].

Barasa E.W. et al. says that "there is a lack of literature on priority setting and resource allocation (PSRA) practices in hospitals, particularly in low and middle-income countries". They made case study and performed research in two public hospitals in coastal Kenya. Data were collected through a combination of:

- in-depth interviews of national-level policymakers,

- hospital managers,

- frontline practitioners in the case study hospitals,

- a review of documents such as hospital plans and budgets,

- parsing of meetings and accounting records,

- non-participant observations of PSRA practices over a period of seven months.

During the investigation, they apply a CAS theory to examine the factors that influence PSRA practices [11]. Researchers found that PSRA practices in the case hospitals were influenced by three factors:

1) Inadequate financing level and poorly designed financing arrangements.

2) Limited hospital autonomy and decision space.

3) Inadequate management and leadership capacity in the hospital.

The case study hospitals showed properties of CAS with multiple interacting agents that reside in a dynamic environment. Problems with process "hardware" (resource scarcity) and "software" (including PSRA guidelines limiting space for hospital decision-making and poor leadership skills) contributed to the proliferation of undesirable properties. The capacity of hospitals to set priorities should be strengthened in all aspects of the organizational structure of the hospital:

- Interventions - should recognize that hospitals are CAS, rather than rectifying isolated aspects of the system.

- Hospital administration - should effort to create conditions for productive emergence [11].

Despite more than two decades of global experience and research on implementation of health systems, progress in integrated care has been limited. Canadian scientists suggested that part of the problem might be how we conceptualize the integration process and the complex systems that contribute organized treatment. The purpose of study, which they published, is to contribute to the discussion on the importance and utility of an integrated care perspective on CAS. The findings of the author indicate that integration is challenged by system complexity, weak ties and poor alignment between professionals and organizations, lack of funding incentives to support collaborative work, and a bureaucratic environment based on a management command and control approach. They identified several CAS characteristics in data, using a CAS framework, including:

- diverse,

- interdependent,

- semi-autonomous actors,

- embedded co-evolutionary systems,

- emerging behaviors,

- non-linearity,

- self-organizing capacity.

One possible explanation for the lack of systemic improvement in processes is that none have been able to treat the healthcare system as complex-adaptive. The data suggest that future implementation efforts need to be anchored from a CAS perspective and focus on building the self-organizing potential of the system. Researchers conclude that integrating care requires policies and management practices that encourage system understanding, relationship building, and information sharing and consider change as an ongoing learning process rather than a sequence of programmatic steps [12].

These are just a few examples that prove the importance and benefits of CAS in healthcare organizations and we believe there will be more and more such studies around the world. 


\section{Complex adaptive systems in nursing practice}

It is also important to pay attention to the organization of the work of nurses. Systems are living entities rather than multi-part machines. The U.S. author notes that machines can be fixed with a replaceable part, while an organic system as a whole need to be looked after. The nurse must therefore always keep in mind the larger picture when caring for the individual patient and expect to be disturbed by unexpected possibilities. The incorporation of complexity science concepts into the practice of nursing leadership encourages new ways of thinking about organizations and is a natural model for the ideals and expected results of skilled nursing [13].

Hodiamont F. et al. says that in palliative care (PC) the definition of complexity can be used to describe the nature of the patient's condition and the nature of the specific needs and treatment requirements. Nevertheless, the term or definition for its specific application in PC is not clearly defined and operationalized. A care situation in PC is characterized by mutual, nonlinear relationships and uncertainties as a complex problem [14]. Dealing with complex problems requires methods to solve problems that are adapted for specific situations. CAS theory provides a framework for defining challenges and solutions. A PC situation's CAS consists of three subsystems: patient, social system and team. The "system patient" agents are delegated to additional patient-level subsystems: physical, psycho-spiritual, and socio-cultural. The "social system" and the "system team" are made up of social agents that influence the CAS as carriers of characteristics, roles and relationships. Environmental factors from outside the system interact with the situation of care subsystem agents and subsystems interact on all hierarchical system levels and shape the system behavior of a PC situation [14].

The benefits observed and in the neonatal care units work. Advances in neonatal care are allowing for increased infant survival; however, neurodevelopmental complications continue. Using a CAS framework, a broad analysis of the network of agents most influential to vulnerable infants in the neonatal intensive care unit (NICU) is presented: parents, nurse, and organization. By exploring these interconnected relationships and the emergent behaviors, a model of care that increases parental caregiving in the NICU is proposed. Supportive parent caregiving early in an infant's NICU stay has the potential for more sensitive caregiving and enhanced opportunities for attachment, perhaps positively impacting neurodevelopment [15].

Paley J., senior lecturer at UK university, writes that our only choices are modest ones at this, still embryonic, point in the development of what we can call "complex science" with reservations. Modest in the sense that we should not make unrealistic and purely speculative claims in a hurry. She also notes that even humble in the sense that we need to look for CAS at the local level, groups and wards, rather than placing meaningless tags on professional bodies, trusts as a whole. Yet finally, modest in the sense that our observations need to be cautiously empirical. CAS theories can be extended to the health service as well as to other organizations, but only if we can transcend the great gestures and the mistaken belief that complexity theory can be combined with a set of common (and rather outdated) principles [16].

Peter Pype and his colleagues examined the effectiveness of a method to improve the functioning of healthcare teams operating in the area of complexity and to determine its effectiveness in recognizing areas for improving team quality. They used the Organizational Capability Questionnaire (OCQ) of the Complex Adaptive Leadership (CALTM) for this project and decided to examine all members of one palliative care team and palliative care physician in Flanders, Belgium. To conclude, researchers write that this questionnaire is a viable and useful tool for assessing the day-to-day activities of the palliative care teams and can be used to identify areas for improving quality. Article authors also note that the CALTM OCQ is a promising method for assessing the functioning of any health care team. A group discussion on the questionnaire scores can serve as a starting point for identifying targets for initiatives to improve quality [17].

In summary, we can note that CAS occupy an important place in the work of nurses and improve the quality of nursing care. 


\section{Conclusions}

Complex adaptive systems, as a tool, step by step, is taking on an increasingly important role in health care and nursing. We can notice similarities between complex adaptive systems and nursing practice. Both are open, non-linear, dynamic, use interactive agents, feedback loops and multiple variables. Also, both require resilience, collaboration, structure and flow, spontaneity, and engaging the unknown. For this reason, complexity science can be applied to the health service as a tool for quality assessment, process optimization and a better understanding of complex situations. We may notice significant influence in the formation of nursing policies and management. It can also be used to assist nurses in assessing patient needs, optimizing the nursing process, improving teamwork and analyzing learning needs.

Complex adaptive systems theory applications will take a permanent place in the healthcare organizations activities, improve nursing processes and create a better work environment for nursing and job satisfaction.

\section{References}

[1] Clancy T. R., Effken J. A., Pesut D. Applications of complex systems theory in nursing education, research, and practice. Nursing Outlook, Vol. 56, Issue 5, 2008, p. 248-256.

[2] Trinier R., Liske L., Nenadovic V. Critical care nursing: embedded complex systems. Canadian Journal of Critical Care Nursing, Vol. 27, Issue 1, 2016, p. 11-16.

[3] Holden L. M. Complex adaptive systems: concept analysis. Journal of Advanced Nursing, Vol. 52, Issue 6, 2005, p. 651-657.

[4] Heather H. Complexity Theory in Nursing. Independent Nurse, 2014, http://www.independentnurse.co.uk/professional-article/complexity-theory-in-nursing/65669/.

[5] Sturmberg J. P., Martin C. M. Handbook of Systems and Complexity in Health. Springer 2013.

[6] Baranger M. Chaos, Complexity, and Entropy. A Physics Talk for Non-Physicists. Report in New England Complex Systems Institute, Cambridge, MA 02138, USA MIT-CTP-3112, http://static1.squarespace.com /static/5b68a4e4a2772c2a206180a1/t/5bf58df18a922d958275788f/1542819314175/cce.pdf.

[7] Chandler J., Rycroft Malone J., Hawkes C., Noyes J. Application of simplified complexity theory concepts for healthcare social systems to explain the implementation of evidence into practice. Journal of Advanced Nursing, Vol. 72, Issue 2, 2016, p. 461-480.

[8] Pype P., Mertens F., Helewaut F., Krystallidou D. Healthcare teams as complex adaptive systems: understanding team behaviour through team members' perception of interpersonal interaction. BMC Health Services Research, Vol. 18, 2018, p. 570.

[9] Ellis B. Complexity in practice: understanding primary care as a complex adaptive system. Informatics in Primary Care, Vol. 18, 2010, p. 135-140.

[10] Flieger S. P. Implementing the patient-centered medical home in complex adaptive systems: Becoming a relationship-centered patient-centered medical home. Health Care Management Review, Vol. 42, Issue 2, 2017, p. 112-121.

[11] Barasa E. W., Molyneux S., English M., Cleary S. Hospitals as complex adaptive systems: A case study of factors influencing priority setting practices at the hospital level in Kenya. Social Science and Medicine, Vol. 174, 2017, p. 104-112.

[12] Tsasis P., Evans J. M., Owen S. Reframing the challenges to integrated care: a complex-adaptive systems perspective. International Journal of Integrated Care, Vol. 12, 2012, p. 190.

[13] James K. M. G. Incorporating complexity science theory into nursing curricula. Creative Nursing, Vol. 16, Issue 3, 2010, p. 137-142.

[14] Hodiamont F., Junger S., Leidl R., Maier B. O., Schildmann E., Bausewein C. Understanding complexity - the palliative care situation as a complex adaptive system. BMC Health Services Research, Vol. 19, 2019 , p. 157.

[15] D'agata A. L., McGrath J. M. A framework of complex adaptive systems. Advances in Nursing Science, Vol. 39, Issue 3, 2016, p. 244-256.

[16] Paley J. Complex adaptive systems and nursing. Nursing Inquiry, Vol. 14, Issue 3, 2007, p. 233-242.

[17] Pype P., Krystallidou D., Deveugele M., Mertens F., Rubinelli S., Devisch I. Healthcare teams as complex adaptive systems: Focus on interpersonal interaction. Patient Education and Counseling, Vol. 100, 2017, p. 2028-2034. 